\title{
Case Study: Neuropathic Itching Following S3 and S4 Dorsal Root Ganglion Stimulator Trial
}

This article was published in the following Dove Press journal:

Journal of Pain Research

\author{
Natalie Strand (iD) \\ Layth Mahdi ${ }^{2}$ \\ Michael E Schatman (iD ${ }^{3,4}$ \\ Jillian Maloney' \\ Christopher Wie' \\ 'Division of Pain Medicine, Mayo Clinic \\ Arizona, Phoenix, AZ, USA; ${ }^{2}$ Mayo Clinic \\ Alix School of Medicine, Phoenix, AZ, \\ USA; ${ }^{3}$ Department of Diagnostic \\ Sciences, Tufts University School of \\ Dental Medicine, Boston, MA, USA; \\ ${ }^{4}$ Department of Public Health and \\ Community Medicine, Tufts University \\ School of Medicine, Boston, MA, USA
}

Correspondence: Michael E Schatman Tel +I 425 647-4880

Email Michael.Schatman@tufts.edu

\section{Introduction}

Dorsal root ganglion (DRG) stimulation is a novel treatment modality for patients suffering from chronic neuropathic pain. When compared to the traditional and broader acting spinal cord stimulator (SCS) implants, DRG stimulation has been shown to be more effective at treating chronic pain. ${ }^{1}$ However, the literature is mixed regarding associated complications of DRG vs traditional SCS implantation. Some studies demonstrate a decreased incidence of paresthesia in DRG stimulators versus SCS, ${ }^{1}$ while others show an increased risk of developing new neurologic symptoms. ${ }^{2}$ The specific characterization of these neurologic symptoms is also less clear. Herein, we present a case of a 49-year-old female who developed neuropathic itching following a bilateral S4 DRG stimulator trial. We include a literature review that demonstrates this approach as a novel and previously unreported complication of a DRG stimulation trial. This project was deemed exempt from the Mayo Clinic Arizona's institutional review board as it acknowledged that it did not meet the criteria for human subject research. The patient provided informed consent for the case to be published.

\section{Case Report}

A 49-year-old female presented with persistent pain for 2 years consequent to falling onto her lower back and buttocks. Following trigger point injections, epidural steroid injections, sacroiliac joint injections, coccyx injections, and ganglion impar injections, she was ultimately referred for a DRG stimulation trial. The patient underwent uncomplicated placement of bilateral S3 DRG leads. Testing failed to provide coverage over her most painful areas, and leads were immediately removed. Bilateral S4 leads were then placed under fluoroscopic guidance, and testing during the procedure provided evidence of excellent coverage. Overall, she tolerated the procedure well with no apparent complications. Approximately 72 hours after the lead placement, she developed perianal and peri-vulvar itching localized specifically to the S3 and S4 dermatomes. It initially was assumed that the itching might be from the adhesive dressings, yet the itching persisted for days after the leads were removed, was not associated with a topical rash and was not present in the location of dressings. The patient reported excellent pain improvement in response to the trial, although the itching persisted for weeks after the trial leads were removed. She was treated with diphenhydramine to decrease the itching, as she reported allergies to gabapentin and pregabalin. After 4 months, her itching 
had reportedly improved substantially, although continued to be occasionally bothersome. As she did not have a prior history of itching and did not initiate any new medications that are known to cause itching, the new and focal itching was attributed to the DRG trial leads.

Both itch and pain pathways in the nervous system are not completely understood. There are many similarities between itch and pain transmission. Both pain and itching are protective mechanisms for humans, pain to signal injury, and itching to signal any foreign substance on the body (ie insects, parasites). A major difference between pain and itch is that pain causes a withdrawal response while itch causes a scratch response. Additionally, itch is typically confined solely to the skin surface, while pain is not. Similarities include that both acute pain and itch can be protective while chronic pain and itch are not. In both chronic pain and itch, there is spontaneous firing in the nervous system as well as central sensitization. There are two main types of itch: histaminergic and nonhistaminergic. C-fiber neurons transmit histamine-mediated itch. ${ }^{3}$ It is unknown whether there are two independent neural pathways for itch and pain or if they share common pathways. Many of the neurotransmitters involved in pain transmission have also been demonstrated to be involved in the transmission of itch sensation, including tumor necrosis factor, interleukin-1, interleukin-6, Substance P, and prostaglandins. ${ }^{4}$ There are primarily two known neuropathic itch conditions that arise from nerve irritation rather from a particular skin disorder: brachioradial pruritus and notalgia paresthetica. Although the specific mechanism of brachioradial pruritus is not fully understood, it is thought to represent a combination of both cervical nerve irritation (C5-C8) and skin exposure to sunlight. ${ }^{5}$ Notalgia paresthetica presents as itching in the upper thoracic dermatomes (T2-T6) and occasionally involves associated pain. It is thought that nerve irritation causes this syndrome as well. That afferent nerve irritation can cause itch supports the hypothesis that both pain and itch share similar neural pathways. Another known similarity of pain and itch is that activation of the mu-opioid receptors can cause itch. There is also a psychosocial component to both itch and pain in that even thoughts of itch and pain can trigger both responses.

\section{Literature Review}

We conducted a literature review by searching PubMed, dates ranging from 1986-2020, with the key words "neuropathic itch DRG", "neuropathic itch stimulator", "DRG stimulator itch", "DRG simulation complication itch", "DRG stimulator pruritus", "spinal stimulator itch", OR "spinal stimulator pruritus". A total of 123 results were reviewed, and none was related to DRG or spine stimulator placement complications or their relation to itch. A secondary search of "DRG stimulation complications" yielded 137 results, of which 3 papers were reviewed for information concerning reported complications. A pooled analysis by Huygen and colleagues found the most frequently reported complications of DRG stimulation were infections $(5.1 \%)$, lead fractures $(5.9 \%)$, lead migration $(5.9 \%)$, temporary motor stimulation $(4.7 \%)$, dural puncture $(4.3 \%)$ and pain at IPG pocket site $(10.2 \%){ }^{6}$ There was no mention of itch or pruritus as a reported complication. A recent retrospective analysis by Kretzschmar and colleagues determined that their complications were consistent with those that Huygen et al had reported. ${ }^{7}$ Another recent study by Horan and colleagues determined that the most common complications were defective electrodes $(39 \%)$, an inability to replace electrodes $(21 \%)$, migrated electrode $(15 \%)$, electrode fragment left in patients $(12 \%)$, nerve damage $(9 \%)$ and infection $(6 \%){ }^{8}$ Neither of the studies mention itch or pruritus as a possible complication.

Eldabe and colleagues conducted a literature review of complications of traditional spinal cord and peripheral nerve stimulation and noted that hardware complications were more common than biological complications. ${ }^{9}$ In addition, complications concerning neurologic damage were related to major neurologic deficit $(0.25 \%)$, limited motor deficit $(0.14 \%)$, autonomic changes $(0.013 \%)$ and sensory deficit $(0.10 \%)$. There was no mention of complications related to itch or pruritus. A study by Sivanesan and colleagues concluded that use of DRG stimulators may result in an increased risk of developing new neurologic symptoms when compared to traditional spinal stimulators. ${ }^{2}$ However, there was a lack of the characterization of these symptoms, with no mention of itch or pruritus as a complication. Given our literature review, we believe our case is likely the first reported DRG stimulator trial resulting in neuropathic itch as a complication.

\section{Discussion}

Our patient experienced new onset itching isolated to the S3 and S4 dermatomes 72 hours post-sacral DRG SCS trial. In the absence of another etiology for itching such as skin irritation or new medications, the patient was diagnosed with neuropathic itching as a complication of sacral 
DRG stimulator lead placement. Evidence suggests that DRG stimulator placement is associated with an increased incidence of neurologic side effects compared to traditional SCS. One proposed mechanism links causality between new neurologic symptoms and spinal dural puncture secondary to the manipulation of needle, guidewire and DRG lead required for lead placement. ${ }^{2}$ A second mechanism relates the manipulation of various components at the neural foramen leading to nerve compression or trauma. This may explain the increased incidence of neurologic symptoms in DRG versus traditional SCS, which does not require manipulation within the fixed space of the neural foramen. While the thoracic and lumbar DRGs are subpedicular in location, the sacral DRG location differs in that it is either intracanalar or intraforaminal. ${ }^{10}$ At S3 and S4, specifically, one study noted that $100 \%$ of the DRGs were intracanalar, or medial to the medial border of the pedicle in the sacrum. ${ }^{11}$ The relevance of anatomic differences between the sacral DRGs compared to lumbar and thoracic DRG location in regard to adverse effects are currently unclear, however.

Neuropathic itch is not as well studied as neuropathic pain, yet remains clinically important and impactful to patients' qualities of life. There are multiple etiologies for neuropathic itch including nerve fiber compression and generalized fiber degeneration. Nerve fiber compression typically causes itch that is localized to the dermatomal distribution of the nerve, while generalized fiber degeneration can lead to either generalized or localized itch. Our patient's neuropathic itch was localized to the S3 and S4 dermatomes, which is likely clinically consistent with the principle of nerve compression. In addition, because DRG lead placement requires component manipulation within the neuronal foramen, it is likely that our patient's neuropathic itch was secondary to nerve compression at the S3 and S4 foramen. Furthermore, the fact that our patient's neuropathy was characterized as "itching" rather than "pain" was intriguing. This is a complicated topic with multiple theories attempting to explain the arising sensation of itch versus pain. Some of these include spatial contrast, temporal pattern, specificity theory and phenotypic switch of nociceptors. ${ }^{12}$ The spatial contrast theory focuses on the pattern of nerve discharge creating a "mismatch signal" that produces the sensation of itch. The temporal pattern focuses on the frequency of nerve discharge while the specificity theory is concerned with neurons critical for itch, such as GRP-releasing neurons. It is likely that some combination of all of these theories contributes to the sensation of itch and its differentiation from pain.

\section{Author Contributions}

All authors made substantial contributions to conception and design, acquisition of data, or analysis and interpretation of data; took part in drafting the article or revising it critically for important intellectual content; agreed to submit to the current journal; gave final approval of the version to be published; and agree to be accountable for all aspects of the work.

\section{Disclosure}

Dr Michael E Schatman is a research consultant for Firstox and Modoscript. The authors report no other conflicts of interest in this work.

\section{References}

1. Deer TR, Levy RM, Kramer J, et al. comparison of paresthesia coverage of patient's pain: dorsal root ganglion vs. spinal cord stimulation. An accurate study sub-analysis. Neuromodulation. 2019;22(8):930-936. doi:10.1111/ner.12920

2. Sivanesan E, Bicket MC, Cohen SP. Retrospective analysis of complications associated with dorsal root ganglion stimulation for pain relief in the FDA MAUDE database. Reg Anesth Pain Med. 2019;44 (1):100-106. doi:10.1136/rapm-2018-000007

3. Carstens E, Follansbee T, Iodi carstens M. The challenge of basic itch research. Acta Derm Venereol. 2020;100(2):adv00023. doi:10.2340/ 00015555-3343

4. Greaves MW, Wall PD. Pathophysiology of itching. Lancet. 1996;348 (9032):938-940. doi:10.1016/S0140-6736(96)04328-0

5. Yosipovitch G, Carstens E, McGlone F. Chronic itch and chronic pain: analogous mechanisms. Pain. 2007;131(1):4-7. doi:10.1016/j. pain.2007.04.017

6. Huygen FJPM, Kallewaard JW, Nijhuis H, et al. Effectiveness and safety of dorsal root ganglion stimulation for the treatment of chronic pain: a pooled analysis. Neuromodulation. 2020;23(2):213-221. doi:10.1111/ner.13074

7. Kretzschmar M, Reining M, Schwarz MA. Three-year outcomes after dorsal root ganglion stimulation in the treatment of neuropathic pain after peripheral nerve injury of upper and lower extremities. Neuromodulation. 2020. doi:10.1111/ner.13222

8. Horan M, Jacobsen AH, Scherer C, et al. Complications and effects of dorsal root ganglion stimulation in the treatment of chronic neuropathic pain: a nationwide cohort study in Denmark. Neuromodulation. 2020. doi:10.1111/ner.13171

9. Eldabe S, Buchser E, Duarte RV. Complications of spinal cord stimulation and peripheral nerve stimulation techniques: a review of the literature. Pain Med. 2016;17(2):325-336. doi:10.1093/pm/ pnv025

10. Esposito MF, Malayil R, Hanes M, Deer T. Unique characteristics of the dorsal root ganglion as a target for neuromodulation. Pain Med. 2019;20(Supplement_1):S23-S30. doi:10.1093/pm/pnz012

11. Ebraheim NA, Lu J. Morphometric evaluation of the sacral dorsal root ganglia. A cadaveric study. Surg Radiol Anat. 1998;20 (2):105-108.

12. Steinhoff M, Oaklander AL, Szabó IL, Ständer S, Schmelz M. Neuropathic itch. PAIN. 2019;160(Suppl 1):S11-S16. doi:10.1097/j. pain.0000000000001551 


\section{Publish your work in this journal}

The Journal of Pain Research is an international, peer reviewed, open access, online journal that welcomes laboratory and clinical findings in the fields of pain research and the prevention and management of pain. Original research, reviews, symposium reports, hypothesis formation and commentaries are all considered for publication. The manuscript management system is completely online and includes a very quick and fair peer-review system, which is all easy to use. Visit http:// www.dovepress.com/testimonials.php to read real quotes from published authors. 